\title{
Single-position prone lateral approach: cadaveric feasibility study and early clinical experience
}

\author{
Jakub Godzik, MD, Ifije E. Ohiorhenuan, MD, PhD, ${ }^{1}$ David S. Xu, MD, ${ }^{2}$ \\ Bernardo de Andrada Pereira, MD, ${ }^{1}$ Corey T. Walker, MD, ${ }^{1}$ Alexander C. Whiting, MD, ${ }^{1}$ \\ Jay D. Turner, MD, PhD, ${ }^{1}$ and Juan S. Uribe, MD ${ }^{1}$
}

\begin{abstract}
1Department of Neurosurgery, Barrow Neurological Institute, St. Joseph's Hospital and Medical Center, Phoenix, Arizona; and ${ }^{2}$ Neurosurgery Department, Houston Methodist Hospital, Houston, Texas
\end{abstract}

OBJECTIVE Lateral lumbar interbody fusion (LLIF) is a useful minimally invasive technique for achieving anterior interbody fusion and preserving or restoring lumbar lordosis. However, achieving circumferential fusion via posterior instrumentation after an LLIF can be challenging, requiring either repositioning the patient or placing pedicle screws in the lateral position. Here, the authors explore an alternative single-position approach: LLIF in the prone lateral (PL) position.

METHODS A cadaveric feasibility study was performed using 2 human cadaveric specimens. A retrospective 2-center early clinical series was performed for patients who had undergone a minimally invasive lateral procedure in the prone position between August 2019 and March 2020. Case duration, retractor time, electrophysiological thresholds, implant size, screw accuracy, and complications were recorded. Early postoperative radiographic outcomes were reported.

RESULTS A PL LLIF was successfully performed in 2 cadavers without causing injury to a vessel or the bowel. No intraoperative subsidence was observed. In the clinical series, 12 patients underwent attempted PL surgery, although 1 case was converted to standard lateral positioning. Thus, 11 patients successfully underwent PL LLIF (89\%) across 14 levels: L2-3 (2 of 14 [14\%]), L3-4 (6 of 14 [43\%]), and L4-5 (6 of 14 [43\%]). For the 11 PL patients, the mean ( \pm SD) age was $61 \pm 16$ years, mean BMI was $25.8 \pm 4.8$, and mean retractor time per level was $15 \pm 6$ minutes with the longest retractor time at $L 2-3$ and the shortest at $L 4-5$. No intraoperative subsidence was noted on routine postoperative imaging.

CONCLUSIONS Performing single-position lateral transpsoas interbody fusion with the patient prone is anatomically feasible, and in an early clinical experience, it appeared safe and reproducible. Prone positioning for a lateral approach presents an exciting opportunity for streamlining surgical access to the lumbar spine and facilitating more efficient surgical solutions with potential clinical and economic advantages.

https://thejns.org/doi/abs/10.3171/2020.6.FOCUS20359

KEYWORDS lateral lumbar interbody fusion; lateral transpsoas; minimally invasive surgery; prone lateral

$\mathrm{T}$ HE minimally invasive lateral lumbar interbody fusion (LLIF) approach to treat degenerative spinal conditions has grown in popularity. Studies have demonstrated satisfactory patient outcomes and appropriate restoration of lordosis, with an acceptable safety profile., ${ }^{1,2}$ For maximal stability, interbody placement is supplemented with pedicle screw fixation to achieve circumferential fixation and fusion. ${ }^{3,4}$

However, a logistical drawback to the LLIF approach is a reliance on multiple patient positions during surgery for interbody access. Particularly, the LLIF approach may require lateral decubitus positioning of the patient for in- terbody access first, followed by prone positioning for percutaneous screw placement. Recently, surgeons have been interested in pursuing single-position surgery to reduce surgical time and to improve the efficiency of surgical flow by reducing the number of repositioning episodes for the patient. ${ }^{5-7}$

Despite some adoption of this technique by neurosurgeons, data are limited regarding the success of singleposition surgery with the patient in a lateral position. Early studies have suggested decreased accuracy with the lateral placement of bilateral pedicle screws, 7,8 although other authors have noted reduced operative times. ${ }^{5,9,10}$ Several au-

ABBREVIATIONS LLIF = lateral lumbar interbody fusion; $\mathrm{PL}=$ prone lateral; $\mathrm{SL}=$ standard lateral.

SUBMITTED April 29, 2020. ACCEPTED June 16, 2020.

INCLUDE WHEN CITING DOI: 10.3171/2020.6.FOCUS20359. 
thors have described challenges in placing pedicle screws with the patient in the lateral decubitus position because of the nonstandard technique, as well as logistical and technical challenges. ${ }^{5,8-11}$

In the current study, we introduce a variation of the standard lateral (SL) decubitus positioning for lateral transpsoas interbody fusion-prone lateral (PL) positioning - to facilitate single-position access to lumbar fusion. To explore this alternate patient positioning, we performed a cadaveric feasibility study and reviewed the early clinical experience with consecutive LLIF cases performed with the patient in the PL position at our institution.

\section{Methods \\ Cadaveric Study Design}

A cadaveric feasibility study was performed using 2 fresh cadaveric dissections (whole torso, 1 male and 1 female, ages unknown) to determine the feasibility of the PL technique for lumbar interbody fusion. Plain radiographs were taken, and specimens with any obvious radiographic flaws, visible flaws, or flaws noted on patient history (metastatic disease, osteophytes, disc collapse) were excluded. Radiographic localization was performed to identify appropriate levels, with interbody fusion performed at all accessible levels. A skin incision was made, and a standard blunt dissection was performed along the retroperitoneal space to approach the spine using a lateral transpsoas corridor. Retractor docking and discectomy were performed at accessible levels (L1-2 to L4-5 level). The location of the psoas muscle relative to the disc space along with the location of the L4-5 disc space relative to the iliac crest was considered. After completion of the surgical intervention, a dissection was performed to inspect the thecal sac and large vessels for evidence of injury; the psoas muscle was also inspected for the location of dilation in relation to the lumbar plexus. Radiographs were analyzed for endplate damage and interbody positioning at each intervertebral level.

\section{Clinical Study Design}

A 2-center retrospective consecutive case series was conducted after approval from each center's respective institutional review board for human research. Patients had provided standard informed consent for the surgical procedures performed; no additional informed consent for this study was required because of its retrospective nature. Inclusion criteria included adult patients (age $\geq 18$ years) with single-level or multilevel degenerative spinal disease who had undergone circumferential LLIF with percutaneous screws in the prone position between August 2019 and March 2020.

Demographic and medical data, LLIF location, cage angulation (degrees), fusion length (levels), surgical details (estimated blood loss, operative duration), and complication profile were collected from medical records. Retractor time, as well as anterior and posterior electrophysiological thresholds on first dilator use, was recorded. Spinopelvic parameters were collected from standing radiographs taken preoperatively and immediately after the surgery. The occurrence of intraoperative subsidence was reviewed on postoperative radiographs and $\mathrm{CT}$ imaging. Pre- and postoperative quality-of-life outcome scores, such as those on the Oswestry Disability Index and visual analog scale for back pain, were collected.

\section{Surgical Technique \\ Positioning}

The patient is positioned prone on a standard Jackson table for the LLIF (Fig. 1A). Hip pads are placed at the posterior-superior iliac spine or slightly below to maximize lordosis. As with lateral decubitus positioning, ${ }^{12}$ adhesive tape is used to stabilize the patient on the table (Fig. 1A and B). However, it is critical to use a contralateral bolster to provide tension against the lumbar spine during graft placement (Fig. 1B) akin to table breaking or placement of a roll to facilitate crest and rib separation, particularly for patients with a high iliac crest. ${ }^{13}$ Taping is used across the chest and at the level of the pelvis to provide stability; tape is then placed on the lateral chest wall (aimed superiorly) and iliac crest (aimed posteriorly) to further separate ribs and iliac crest, as well as to tension the skin for easier soft-tissue dissection on the side of approach. Notably, our choice of surgical approach is similar to SL approaches. Our preference is from the side of concavity or the side with more amenable psoas anatomy; if there is equipoise with respect to approach side, our preference is the right side because of the vessel anatomy.

\section{Imaging}

Standard fluoroscopy with the C-arm is used to identify the level of interest. Use of CT navigation is an alternative for LLIF. ${ }^{14}$ First, an anteroposterior image is obtained, and the Jackson table is adjusted (tilt and Trendelenburg) to facilitate an orthogonal position. Next, a lateral radiograph is obtained, and the bed is arranged so that the level of interest is orthogonal with the facing wall. An incision is marked on the lateral side in a standard fashion (Fig. 1C). ${ }^{12}$

\section{Operative Procedure}

The operative field is draped to include the posterior aspect of the lumbar spine, bilateral posterior-superior iliac spine (intended for neuronavigation tracker placement), and lateral access corridor (Fig. 1D). A retroperitoneal transpsoas approach is performed to bluntly access the lateral aspect of the spine in a standard fashion, ${ }^{12,13}$ with the surgeon seated on a stool on the ipsilateral side of the surgical approach (Fig. 2A). The dilator is then placed perpendicular to the spine of the prone patient (Fig. 2B), respecting the standard "safe zones" previously described ${ }^{15}$ with radiographic confirmation using lateral fluoroscopy. Sequential dilation through the psoas muscle is performed (Fig. 2C), and electromyography thresholds are then obtained in a directional manner in a standard fashion, ${ }^{13}$ respecting the previously described threshold limits. ${ }^{16}$ Notably, in our experience, the psoas muscle appears to be displaced posteriorly compared to its position in the lateral decubitus position. This resulted in a tendency for more posterior docking despite acceptable electromyography threshold values. The retractor is then docked and attached to the retractor arm (Fig. 2D); notably the retractor 


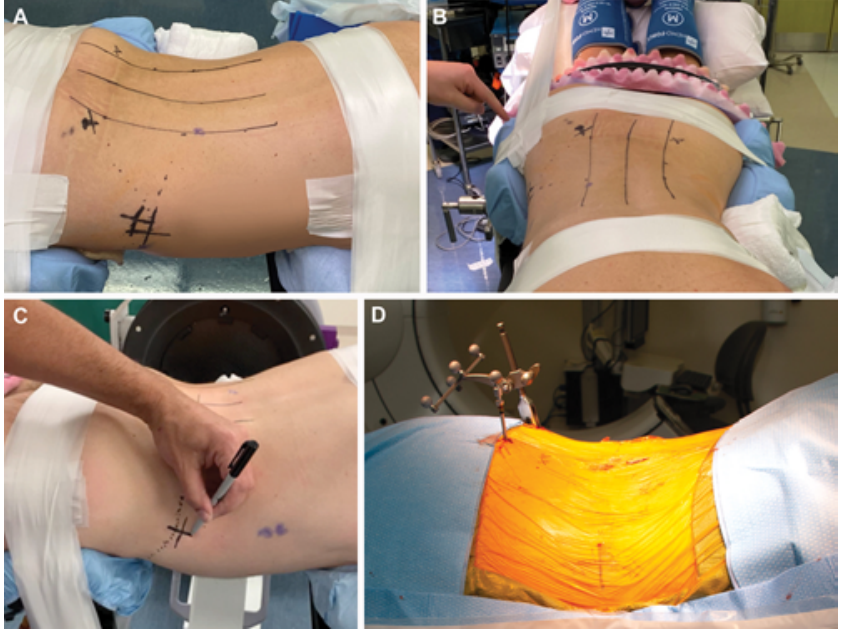

FIG. 1. Patient positioning for a prone LLIF with pedicle screw placement. A: Patient positioning on the Jackson table with adhesive tape applied across the hips and chest, with tape used to stretch skin on the ipsilateral side. B: Contralateral bolster positioned to facilitate slight bend and to separate ribs from the iliac crest. C: Use of fluoroscopy to identify the level of interest and mark skin incision. D: Draping of the operative field with the percutaneous navigation tracker in place.

arm is attached to the bed on the ipsilateral side. Retractor positioning is then verified with lateral and anteroposterior fluoroscopy. Next, a standard wide discectomy with contralateral annulotomy is performed (Fig. 2E). ${ }^{12,13}$ Implant trialing and placement is then performed in a standard fashion, ${ }^{12}$ with care not to over-distract and cause endplate fracture (Fig. 2F). ${ }^{17}$ Implants are selected on the basis of patient anatomical factors and bone quality; implants typically provide $10^{\circ}-15^{\circ}$ of lordosis and $8-10 \mathrm{~mm}$ in height.

The second stage includes the placement of percutaneous pedicle screws using CT navigation with sentinel fluoroscopic imaging (Fig. 2G). First, the tensioning tape is removed to reduce strain on the soft tissues and decrease curvature of the spine. Next, an iliac crest tracker is placed and intraoperative CT is performed. Navigation is then used in a standard fashion to place percutaneous pedicle screws with anteroposterior fluoroscopic imaging to verify accuracy. ${ }^{18}$

\section{Statistical Analysis}

Data are reported as number (percent) and mean \pm standard deviation. Retractor times and electrophysiological thresholds are expressed as the means for each level. A comparison of preoperative and immediate postoperative radiographs was performed. Statistical significance was established using a threshold of $\mathrm{p}<0.05$. SPSS Statistics for Windows (version 24; IBM Corp.) was used for all analyses.

\section{Results \\ Cadaveric Feasibility Study}

Two cadaveric specimens were used to assess the PL approach to the spine. With the cadaveric torsos in the prone position, access to the L1-2 through L4-5 levels was
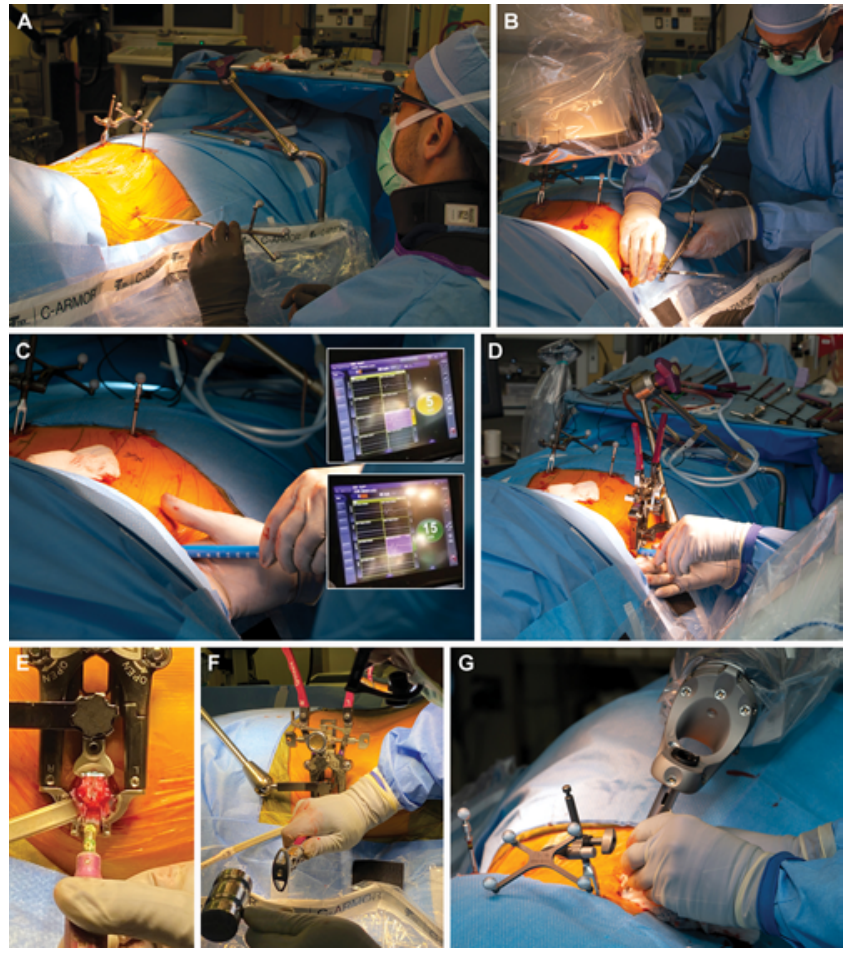

FIG. 2. Operative steps. A: Patient in the prone position with the surgeon in a seated position. B: Initial dilator placement with the patient prone. C: Use of sequential dilation through the psoas muscle with the use of electromyography, with higher thresholds inferiorly and lower values superiorly. D: Retractor placement. E: Visualization of the disc and placement of the anterolateral ligament retractor to perform a wide annulotomy. F: Interbody placement once the discectomy is complete. G: Pedicle screw placement using CT navigation and robotically assisted guidance.

possible without the need for angled instruments. Access to L1-2 required dilation between T11 and T12 ribs. No vascular or intestinal injury was appreciated on inspection, nor did incidental anterolateral ligament release occur. Implant sizes ranged from 8 to $12 \mathrm{~mm}$ in height and 55 to $60 \mathrm{~mm}$ in length. Postintervention radiographic inspection did not reveal iatrogenic endplate damage. Interbody placement was at designated safe working zones (zone 3 at L1-2 through L3-4; zone 3 at L4-5): ${ }^{15}$ dissection demonstrated dilation through the psoas muscle anterior to the lumbar plexus in both cases (Fig. 3).

\section{Clinical Case Series}

Twelve patients meeting the study inclusion criteria underwent attempted single-position PL interbody placement with standard prone percutaneous pedicle screw fixation; 11 patients successfully underwent the PL surgery and 1 patient was transitioned from the PL to SL position for the final surgery. The mean age of patients undergoing PL surgery was $61 \pm 16$ years, and 6 of the 11 patients $(54 \%)$ were women. The diagnoses for PL interbody placement among the $11 \mathrm{PL}$ patients were spondylolisthesis (7 of 11 [64\%]), degenerative disc disease (3 of 11 [27\%]), and adjacent segment disease (1 of 11 [9\%]). The mean preoperative radiographic parameters for the $11 \mathrm{PL}$ patients were a 


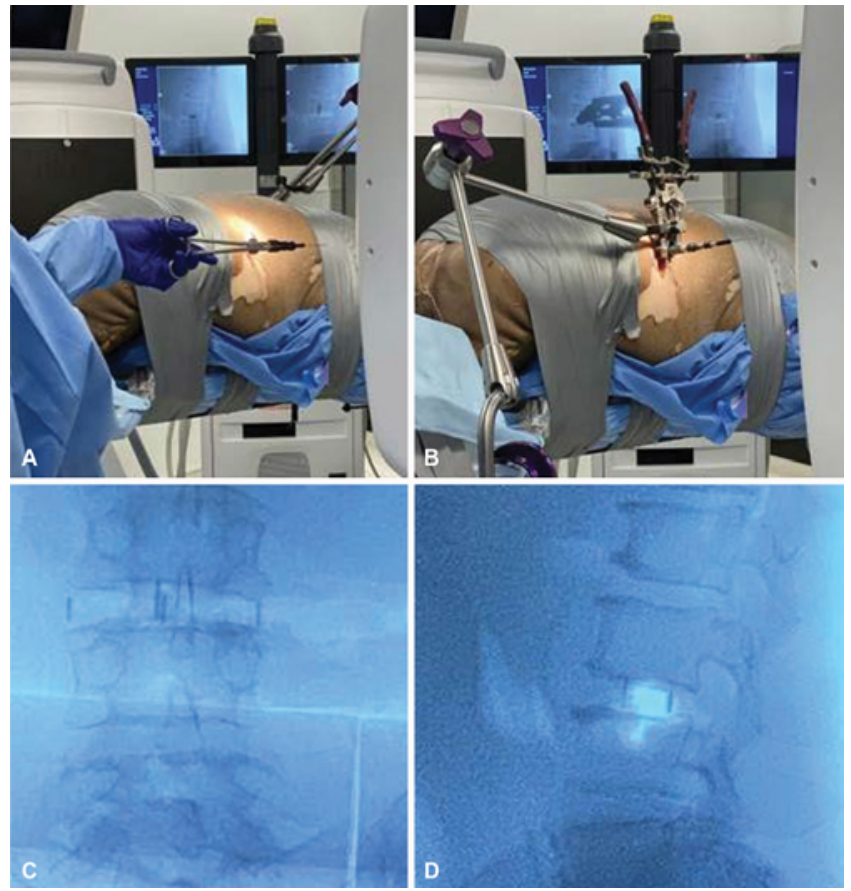

FIG. 3. Cadaveric procedure including prone positioning of the dilator (A) and retractor (B). Anteroposterior (C) and lateral (D) radiographs showing interbody placement.

pelvic incidence of $52^{\circ} \pm 15^{\circ}$, lumbar lordosis of $52^{\circ} \pm 14^{\circ}$, pelvic incidence-lumbar lordosis mismatch of $4^{\circ} \pm 11^{\circ}$, and sagittal vertical axis of $3.1 \pm 1.9 \mathrm{~cm}$ (Table 1). The mean weight of the $11 \mathrm{PL}$ patients was $76.9 \pm 23 \mathrm{~kg}$, and the mean BMI was $25.8 \pm 4.8$; the patient who was unable to undergo PL surgery had a body weight of $108 \mathrm{~kg}$ and BMI of 35 .

\section{Surgical Details}

Surgical results are presented for the 11 patients who successfully underwent the PL approach. Lateral transpsoas interbody fusion with patients in the PL position was performed at 14 levels: L2-3 (2 of 14), L3-4 (6 of 14), and L4-5 (6 of 14). The approach was performed from the right side in 8 of 11 patients $(73 \%)$ and from the left side in 3 of 11 patients (27\%). The most commonly used cage was $10^{\circ}$ ( 9 of 14 levels). The mean case duration was $175 \pm 101$ minutes with a mean estimated blood loss of $19 \pm 14 \mathrm{ml}$. The mean retractor time per level was $15 \pm 6$ minutes. The mean electrophysiological threshold values are reported in Table 2. Additional percutaneous pedicle screw fixation was performed across 13 levels in 10 patients. The mean overall fluoroscopy time was $267 \pm 98$ seconds, with a mean radiation emission dose of $171 \pm 60$ mGy. No perioperative subsidence was noted on intraoperative radiographs.

Among all 12 patients, one experienced postoperative urinary retention that resolved on admission. Six patients described transient ipsilateral pain with hip flexion, which resolved before they were discharged from the hospital. Two patients had transient postoperative thigh pain that

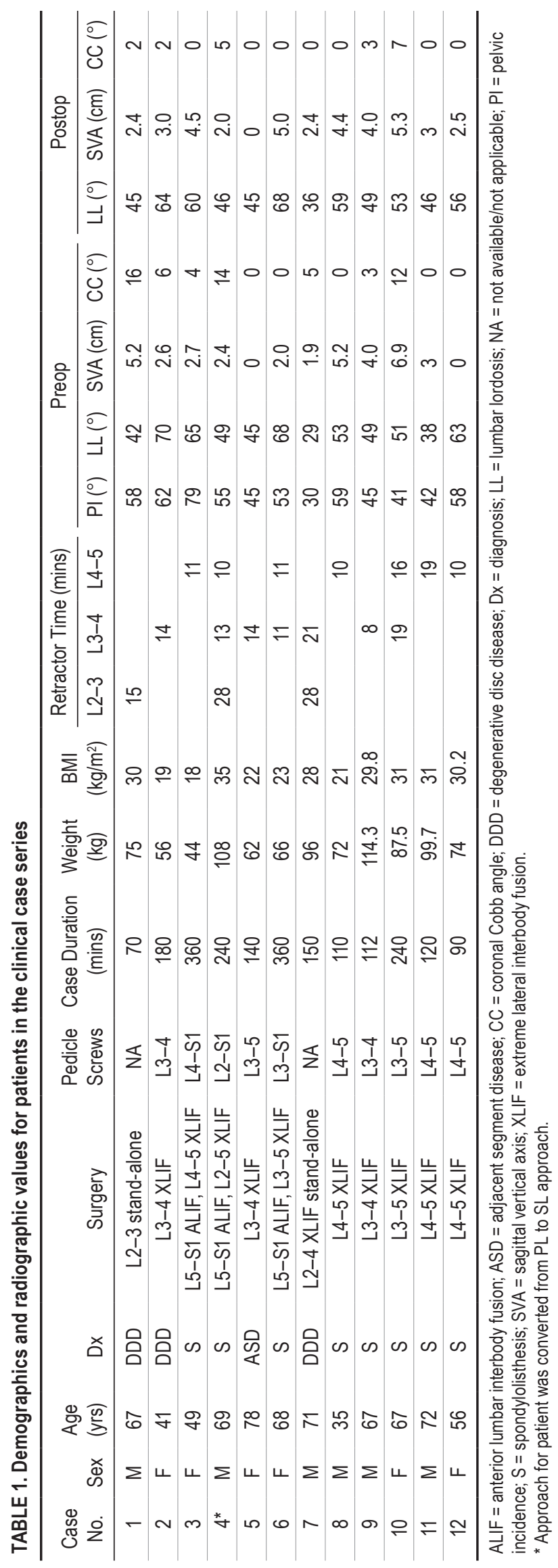


TABLE 2. Mean surgical times and electrophysiological values per level

\begin{tabular}{cccccc}
\hline Level & $\begin{array}{c}\text { No. of } \\
\text { Levels }\end{array}$ & $\begin{array}{c}\text { Retractor } \\
\text { Time } \\
(\text { mins })\end{array}$ & $\begin{array}{c}\text { Anterior } \\
\text { EMG } \\
\text { Threshold } \\
(\mathrm{mA})\end{array}$ & $\begin{array}{c}\text { Posterior } \\
\text { EMG } \\
\text { Threshold } \\
(\mathrm{mA})\end{array}$ & Subsidence \\
\hline L1-2 & 0 & - & - & - & - \\
\hline L2-3 & 2 & $21.5 \pm 9.1$ & $40 \pm 0$ & $32 \pm 11.3$ & 0 \\
\hline L3-4 & 6 & $14.4 \pm 4.9$ & $38 \pm 3$ & $25 \pm 11$ & 0 \\
\hline L4-5 & 6 & $12.8 \pm 3.8$ & $21 \pm 8$ & $12 \pm 9$ & 0 \\
\hline
\end{tabular}

EMG = electromyography

resolved within 7 days. The mean length of stay was $2 \pm$ 2 days.

\section{Outcomes}

The mean follow-up was $59 \pm 29$ days. Early postoperative radiographic values are reported in Table 1 . The mean lumbar lordosis increased to $53^{\circ} \pm 11^{\circ}$, the sagittal vertical axis increased to $3.3 \pm 1.6 \mathrm{~cm}$, and the coronal Cobb angle decreased to $1.3^{\circ} \pm 1.3^{\circ}$. Postoperative MRI and CT imaging demonstrated well-positioned interbodies at all levels, without dorsal to ventral angulation; no immediate postoperative or perioperative subsidence was observed. No lateral or medial pedicle breaches were observed on postoperative CT imaging (0 of 46 pedicle screws) with pedicle screw fixation. The mean preoperative Oswestry Disability Index was $55.1 \pm 30.4$ and improved to $28.5 \pm$ $18.0(\mathrm{p}=0.03)$ after surgery, and the mean preoperative back pain score was $6.0 \pm 2.3$ and improved to $1.6 \pm 0.8$ (p $<0.001)$ at the early follow-up.

\section{Illustrative Case}

A 35-year-old man (case 8) presented with a longstanding history of low-back pain and unilateral radiculopathy that was not responsive to conservative management. Imaging demonstrated an L4 bilateral pars defect with resultant mobile spondylolisthesis (Fig. 4). The patient underwent a prone right-sided LLIF with posterior percutaneous fixation. He was positioned as previously described for a PL approach, with a hip pad placed on the contralateral side to provide counterpressure; tape was used to provide a slight opening of the space between the rib cage and iliac crest, along with tensioning of the skin for easier dissection. The first stage involved the lateral interbody fusion; standard dissection was performed through the soft tissues, and care was taken to mobilize the retroperitoneal contents as described in previous studies. The retractor was docked with radiographic verification, and the interbody fusion was performed in $10 \mathrm{~min}-$ utes, 15 seconds. Next, an iliac crest-based intraoperative tracker was placed, and intraoperative CT was performed. Percutaneous pedicle screws were placed with navigation
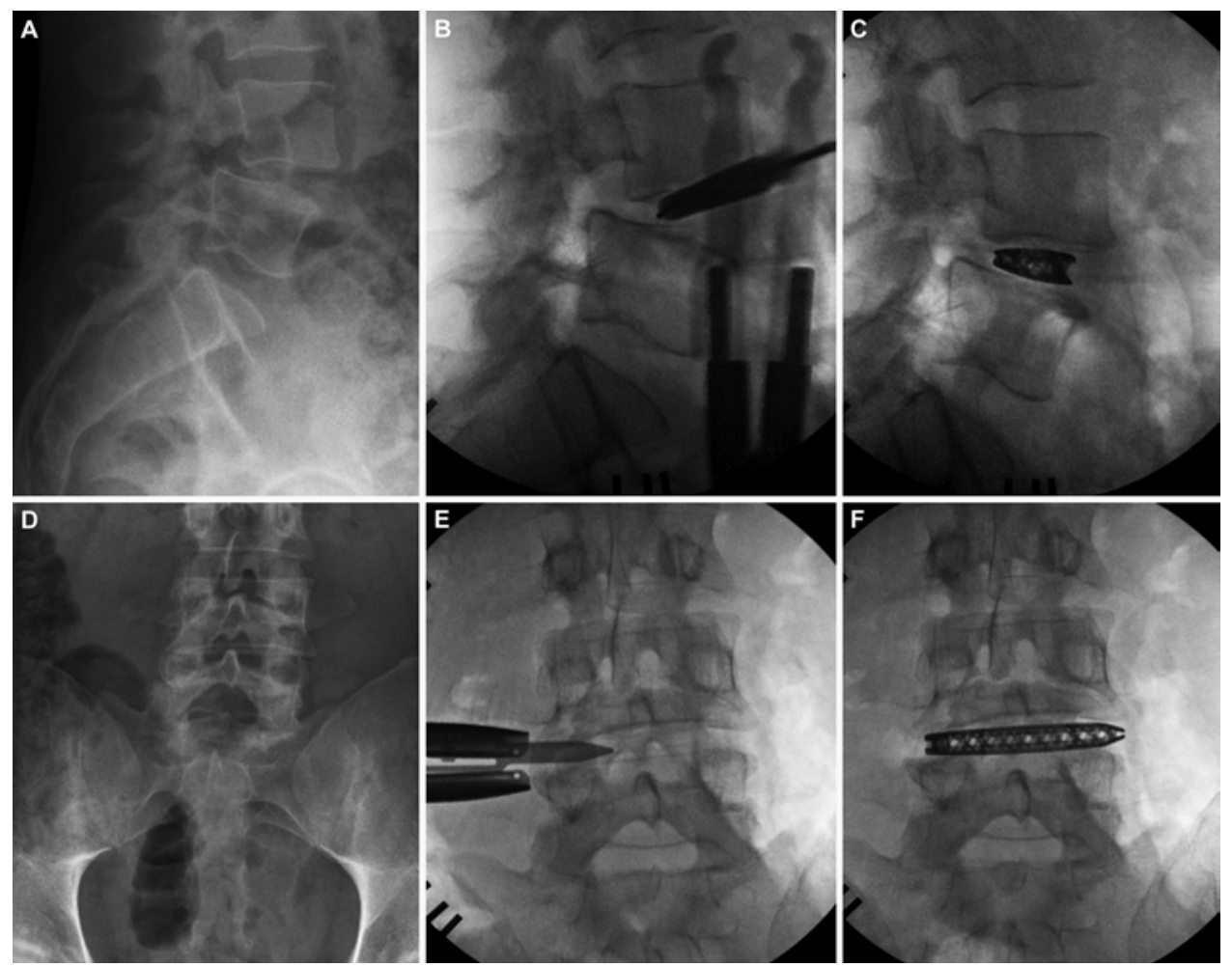

FIG. 4. Case 8. Example of single-position lateral interbody fusion at $L 4-5$. A: Preoperative lateral radiograph demonstrating $L 4-5$ spondylolisthesis. B: Intraoperative lateral radiograph demonstrating dilator placement at zone 2/3 with acceptable electromyography thresholds. C: Intraoperative lateral radiograph demonstrating final titanium implant location. D: Preoperative anteroposterior radiograph. E: Intraoperative anteroposterior radiograph demonstrating docked retractor with shim placement at L4-5. F: Intraoperative anteroposterior radiograph demonstrating placement of the implant across the L4-5 disc space without subsidence. 


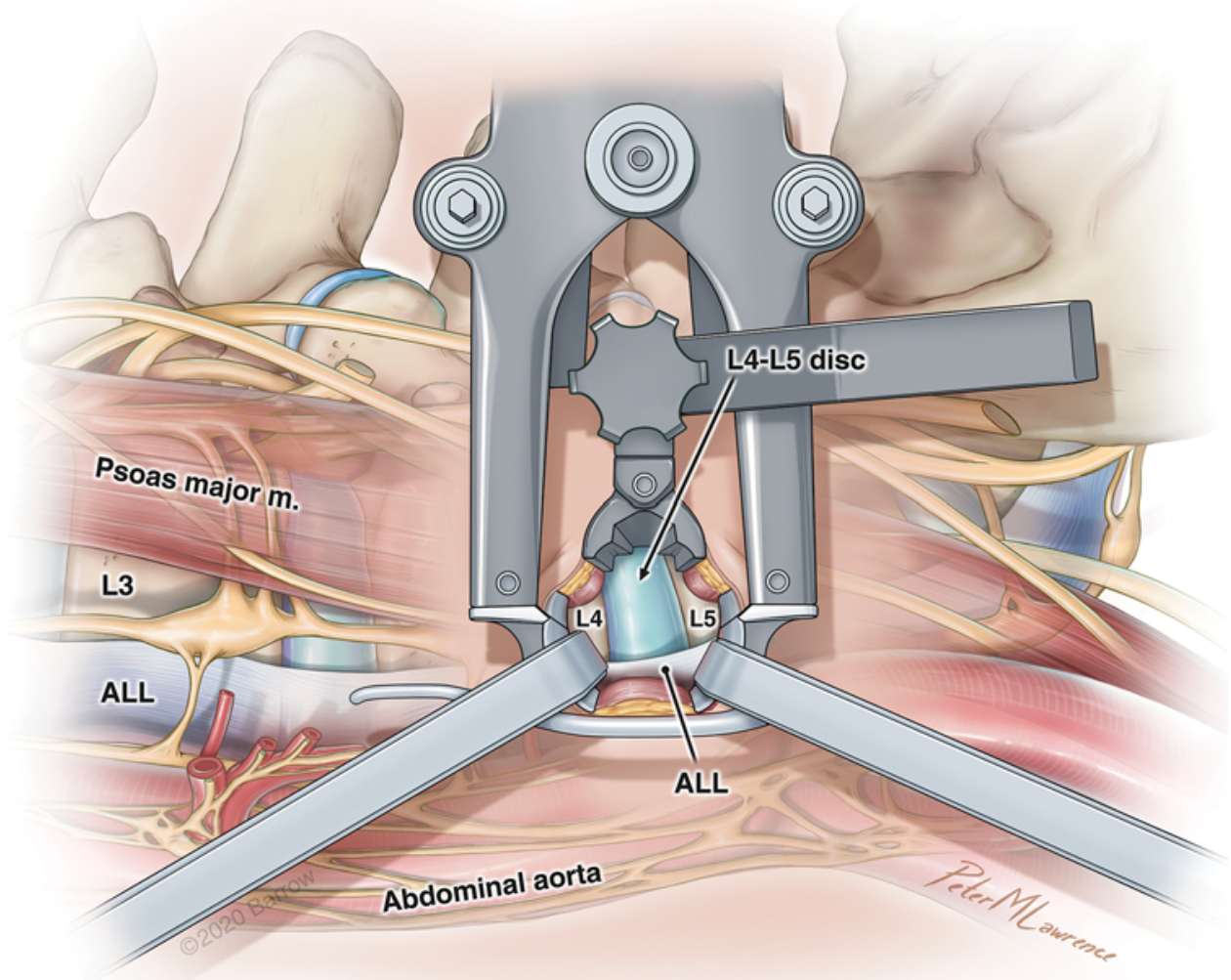

FIG. 5. Illustration of PL retractor placement with relative orientation to the floor and the lumbar plexus. ALL = anterior longitudinal ligament; $\mathrm{m}$. = muscle. Illustration used with permission from Barrow Neurological Institute, Phoenix, Arizona.

assistance and the use of sentinel fluoroscopy. Lastly, reduction of the spondylolisthesis was performed with rod fixation and final tightening.

\section{Discussion}

While the use of the lateral approach corridor to the lumbar spine has outcomes comparable to those for posterior-only approaches such as transforaminal lumbar interbody fusion, SL positioning presents a logistical challenge when attempting circumferential fusion. The lateral singleposition surgery variation facilitates a more streamlined workflow; however, authors have described the technical challenges involved with placing pedicle screws in the lateral position and difficulty when addressing 3 or more interbody fusion levels. ${ }^{8-10}$ We posit that performing the transpsoas approach with patients in the PL position leverages the advantage of the lateral minimally invasive technique, without the need for repositioning, and represents a maximally streamlined approach toward single-position surgery.

In the current paper, we present our findings from a cadaveric feasibility study and the early clinical experience of a single surgeon using this prone single-position technique to address degenerative spinal disease (Fig. 5). We noted successful and effective interbody fusion with PL positioning across 14 levels in 11 patients, along with pedicle screw placement across 13 levels in 10 patients. We aborted the PL approach in 1 patient and converted to an LLIF in the
SL position because the patient's abdominal girth exceeded the maximum capacity of our instruments, although his BMI of 35 was within the range of other patients in our sample. Furthermore, the patient's abdominal girth made it difficult to adequately palpate the psoas muscle during the approach. Given the potential for a colon injury with poorly controlled dilation through the psoas muscle, the decision was made to abort the PL approach in this case because of safety concerns. This may be an interesting phenomenon associated with prone positioning, which, unlike lateral positioning, results in more soft-tissue accumulation between the skin and lumbar spine. Notably, in subsequent cases, we were able to overcome soft-tissue concerns (with an enlarged incision and greater dissection); however, in the future, we will give more attention to abdominal girth and adipose distribution on preoperative examination.

Several studies have described a single-position approach to minimally invasive surgery for the lumbar spine with patients in the lateral decubitus position. Blizzard and Thomas $^{7}$ performed a retrospective review of 72 consecutive cases of 1- to 2-level LLIF or oblique LLIF with bilateral pedicle screw fixation. The authors noted an average screw placement time of $5.9-\mathrm{min} / \mathrm{screw}$, with a $5.1 \%$ breach rate and $2.8 \%$ reoperation rate for symptomatic malpositioned screws. In particular, these authors noted a challenge with the L5-S1 level, specifically the S1 level, possibly due to decreased tactile feedback or challenges with patient positioning. Hiyama et al. ${ }^{9}$ noted that single-position surgery is a feasible modification to the standard workflow. They also 
TABLE 3. Relative advantages of prone and lateral positioning for lumbar interbody fusion procedures

\begin{tabular}{lcc}
\hline \multicolumn{1}{c}{ Advantage } & Prone & Lateral \\
\hline Navigation & Slight advantage & Equivalent \\
\hline $\begin{array}{l}\text { Direct decompression or } \\
\text { facetectomies (simultaneous) }\end{array}$ & Slight advantage & No advantage \\
\hline Screw placement & $\begin{array}{l}\text { Substantial } \\
\text { advantage }\end{array}$ & Equivalent \\
\hline Positional lordosis & Slight advantage & Equivalent \\
\hline ALIF (simultaneous) & No advantage & Equivalent \\
\hline Patient obesity & Equivalent & Slight advantage \\
\hline
\end{tabular}

$\mathrm{ALIF}=$ anterior lumbar interbody fusion .

noted that lateral positioning for pedicle screw placement has a limited working corridor between the patient and the C-arm and advocated the use of guidewire-less screw systems. Sellin et al. ${ }^{8}$ noted a $14 \%$ lateral breach rate in 4 patients who had undergone single-position surgery with lateral percutaneous pedicle screw placement; the authors noted that all breaches occurred on the "down side" and could be attributed to poor patient positioning. The authors also reported that all breaches required a return to surgery for repositioning and noted that in 1 case bilateral pedicle screws were planned but could not be placed because of challenges with patient positioning. Ziino et al. ${ }^{19}$ described a shorter time with single-position surgery than that with dual-position surgery across 42 patients but also reported a higher likelihood of unilateral screws in single-position surgery (19\% of patients) than in dual-position surgery $(0 \%$ of patients). These authors also reported a $4.7 \%$ rate of return to surgery for screw repositioning (2 of 42 patients). As a result of the nonstandard positioning associated with lateral single-position surgery, several authors have described a decrease in surgical time but significant technical challenges with screw placement. . $^{-10}$

The alternate approach, prone-position surgery, was recently described by Lamartina and Berjano ${ }^{20}$ with a comparison between SL decubitus positioning and the prone approach. These authors similarly noted successful implantation in the majority of cases across 7 levels in 7 patients. They reported $133 \pm 26$ minutes for total operative duration, with a mean retractor time of $25.6 \pm 13$ minutes. While we did not measure the prone positioning time or compare it to times for SL decubitus positioning, we similarly observed an anecdotal trend toward shorter setup and positioning times. Similarly, the majority of our surgical cases focused on the L3-4 and L4-5 levels, but we did also operate on L2-3 levels, with an increased duration of retractor exposure likely owing to the slightly more challenging anatomy. However, comparatively, we did not experience any episodes of intraoperative cage subsidence. While we did not evaluate surgical outcomes in the current study, we did note a rate of postoperative hip pain that was similar to that published by Lamartina and Berjano, ${ }^{20}$ which appeared similarly transient. Overall, we agree that such prone positioning presents an attractive option for streamlined surgical solutions.

Theoretical advantages of prone positioning include positional lordosis if needed, the opportunity for direct decompression if needed, and the facilitation of a more natural and easier approach for screw placement (Table 3), along with potential hemostatic benefit, as previously suggested by other authors. ${ }^{11}$ In addition, the prone position presents, in our opinion, more amenable positioning for the use of navigation-the patient appears more secure in the prone position than in the lateral-with greater points of fixation and fewer chances of losing accuracy with rotatory movements of the upper compared to the lower spine; this may provide improved fidelity of navigation and improved accuracy of screw placement. However, the current study is not designed to validate this concept.

Notably, we did not experience any anterior displacement of tools or instrumentation with patients in the prone position, as we had initially expected. Rather, the psoas muscle appears to be shifted posteriorly, possibly because of hip extension when the patient is positioned prone on a Jackson table as compared to hip flexion, which typically occurs when the patient is in the lateral position. Thus, somewhat counterintuitively, with PL positioning the placement of the dilation tube and instrumentation tends to migrate posteriorly when compared to placement in the SL position. In our experience, we noted an anecdotal trend toward higher electromyography thresholds with PL positioning than with SL positioning. Notably, we did not observe any tendency for implants to be placed anteriorly or with a dorsal to ventral angulation on postoperative imaging.

In our view, the PL positioning does not replace the SL positioning for LLIF, but in select patients, it can be a viable alternative that potentially increases efficiency and decreases operative time. We believe PL positioning is indicated in cases in which patient anatomy is amenable and in patients who are not large or morbidly obese. In our experience, it appears that body weight and distribution of adiposity are more critical than the observed BMI. We would recommend that, for more challenging anatomy (e.g., high crest, obesity, or transitional anatomy), surgeons default to the more familiar SL positioning until experience with the PL position is attained. This paper presents the largest clinical series published to date on the PL variant of the lateral approach. Although not the primary objective of the paper, this early experience provisionally demonstrates that this approach is safe, feasible, and reproducible.

\section{Study Limitations}

This study has several key limitations. First, the study has a retrospective design without a comparison group. As a result, we were unable to compare the prone single-position surgery to a lateral decubitus single-position surgery or dual-position alternatives in terms of surgical timing, accuracy, or patient outcome data. However, the primary objective of the study was to describe our initial experience and to complement the existing literature regarding prone positioning. Additionally, we did not perform quantitative or comprehensive measurements of surgical time-particularly time-saving elements that included positioning or time per screw placed. Lastly, our study describes only the short-term perioperative experience and 
complications for patients and does not include extensive long-term patient-level outcomes. While initial postoperative neurological deficits were evaluated, no long-term patient-level outcome data were analyzed. We feel that future prospective studies with larger sample sizes are needed to fine-tune our surgical workflow, optimize lateral access circumferential fusion, and provide an accurate comparison to traditional and other single-position variants for a wide variety of patients (e.g., those with obesity, a high crest, or transitional anatomy).

\section{Conclusions}

Prone positioning for the lateral approach appears to be a viable and exciting option for minimally invasive spine surgery. Although future studies are needed, our initial experience indicates that the prone position is both safe and effective.

\section{Acknowledgments}

We thank the staff of Neuroscience Publications at Barrow Neurological Institute for assistance with manuscript preparation.

\section{References}

1. Louie PK, Haws BE, Khan JM, et al. Comparison of standalone lateral lumbar interbody fusion versus open laminectomy and posterolateral instrumented fusion in the treatment of adjacent segment disease following previous lumbar fusion surgery. Spine (Phila Pa 1976). 2019;44(24):E1461-E1469.

2. Smith JS, Shaffrey E, Klineberg E, et al. Prospective multicenter assessment of risk factors for rod fracture following surgery for adult spinal deformity. J Neurosurg Spine. 2014; 21(6):994-1003.

3. Pimenta L, Turner AW, Dooley ZA, et al. Biomechanics of lateral interbody spacers: going wider for going stiffer. ScientificWorldJournal. 2012;2012:381814.

4. Godzik J, Martinez-Del-Campo E, Newcomb AGUS, et al. Biomechanical stability afforded by unilateral versus bilateral pedicle screw fixation with and without interbody support using lateral lumbar interbody fusion. World Neurosurg. 2018;113:e439-e445.

5. Hiyama A, Sakai D, Sato M, Watanabe M. The analysis of percutaneous pedicle screw technique with guide wire-less in lateral decubitus position following extreme lateral interbody fusion. J Orthop Surg Res. 2019;14(1):304.

6. Quiceno E, Hartman C, Godzik J, et al. Single position spinal surgery for the treatment of grade II spondylolisthesis: a technical note. J Clin Neurosci. 2019;65:145-147.

7. Blizzard DJ, Thomas JA. MIS single-position lateral and oblique lateral lumbar interbody fusion and bilateral pedicle screw fixation: feasibility and perioperative results. Spine (Phila Pa 1976). 2018;43(6):440-446.

8. Sellin JN, Brusko GD, Levi AD. Lateral lumbar interbody fusion revisited: complication avoidance and outcomes with the mini-open approach. World Neurosurg. 2019;121:e647-e653.

9. Hiyama A, Katoh H, Sakai D, et al. Comparison of radiological changes after single- position versus dual- position for lateral interbody fusion and pedicle screw fixation. $B M C$ Musculoskelet Disord. 2019;20(1):601.

10. Ziino C, Arzeno A, Cheng I. Analysis of single-position for revision surgery using lateral interbody fusion and pedicle screw fixation: feasibility and perioperative results. $J$ Spine Surg. 2019;5(2):201-206.
11. Ouchida J, Kanemura T, Satake K, et al. Simultaneous singleposition lateral interbody fusion and percutaneous pedicle screw fixation using $\mathrm{O}$-arm-based navigation reduces the occupancy time of the operating room. Eur Spine J. 2020;29(6): 1277-1286.

12. Yen CP, Uribe JS. Procedural checklist for retroperitoneal transpsoas minimally invasive lateral interbody fusion. Spine (Phila Pa 1976). 2016;41(suppl 8):S152-S158.

13. Ozgur BM, Aryan HE, Pimenta L, Taylor WR. Extreme Lateral Interbody Fusion (XLIF): a novel surgical technique for anterior lumbar interbody fusion. Spine J. 2006;6(4):435-443.

14. Park P. Three-dimensional computed tomography-based spinal navigation in minimally invasive lateral lumbar interbody fusion: feasibility, technique, and initial results. Neurosurgery. 2015;11(suppl 2):259-267.

15. Uribe JS, Arredondo N, Dakwar E, Vale FL. Defining the safe working zones using the minimally invasive lateral retroperitoneal transpsoas approach: an anatomical study. $J$ Neurosurg Spine. 2010;13(2):260-266.

16. Uribe JS, Vale FL, Dakwar E. Electromyographic monitoring and its anatomical implications in minimally invasive spine surgery. Spine (Phila Pa 1976). 2010;35(26)(suppl):S368S374.

17. Wewel JT, Hartman C, Uribe JS. Timing of lateral lumbar interbody subsidence: a review of exclusive intraoperative subsidence. World Neurosurg. 2020;137:e208-e212.

18. Kim TT, Drazin D, Shweikeh F, et al. Clinical and radiographic outcomes of minimally invasive percutaneous pedicle screw placement with intraoperative CT (O-arm) image guidance navigation. Neurosurg Focus. 2014;36(3):E10.

19. Ziino C, Konopka JA, Ajiboye RM, et al. Single position versus lateral-then-prone positioning for lateral interbody fusion and pedicle screw fixation. J Spine Surg. 2018;4(4):717-724.

20. Lamartina $C$, Berjano P. Prone single-position extreme lateral interbody fusion (Pro-XLIF): preliminary results. Eur Spine J. 2020;29(1)(suppl 1):6-13.

\section{Disclosures}

Dr. Turner is a consultant for NuVasive and SeaSpine. Dr. Uribe is a consultant for, receives royalties from, and has direct stock ownership in NuVasive and is a consultant for Misonix and SI-BONE.

\section{Author Contributions}

Conception and design: Uribe, Godzik. Acquisition of data: Xu, de Andrada Pereira, Whiting. Analysis and interpretation of data: Uribe, Godzik, Ohiorhenuan. Drafting the article: de Andrada Pereira. Critically revising the article: Walker, Whiting, Turner. Reviewed submitted version of manuscript: Uribe. Statistical analysis: Godzik, Whiting. Administrative/technical/material support: Turner. Study supervision: Uribe, Turner.

\section{Supplemental Information \\ Videos}

Video Abstract. https://vimeo.com/445620664.

\section{Correspondence}

Juan S. Uribe: c/o Neuroscience Publications, Barrow Neurological Institute, St. Joseph's Hospital and Medical Center, Phoenix, AZ.neuropub@barrowneuro.org. 